\title{
smarter medicine: la liste «Top-5» de la SPSG
}

\section{Organisation responsable «smarter medicine»}

\section{Choosing wisely et la gériatrisation de la médecine}

La société américaine de gériatrie a publié, il y a quelque temps, dix recommandations dans le cadre de son initiative choosing wisely. La Société professionnelle suisse de gériatrie (SPSG) souscrit aux recommandations choosing wisely spécifiques à la gériatrie de la société américaine de gériatrie. Il est important qu'elles soient également observées en Suisse, dans toutes les institutions responsables de la prise en charge de personnes très âgées.

Nous vous présentons les cinq premières recommandations dans ce document. L'objectif est de mettre en œuvre les découvertes fondamentales en médecine gériatrique, également dans des institutions n'ayant pas (encore) directement accès à des experts en gériatrie.

1. Ne pas recommander l'alimentation par sonde gastrique percutanée chez les patients présentant une démence sévère; proposer à la place une alimentation assistée par voie orale.

Aider un patient atteint de démence sévère à se nourrir est une méthode au moins aussi bonne que l'alimentation par sonde eu égard au pronostic de survie, de pneumonies d'aspiration, de fonctionnalité et de confort du patient. L'apport nutritif doit avant tout reposer sur l'alimentation. L'alimentation par sonde est associée à un risque d'agitation, à une augmentation de l'utilisation de moyens de contention physique et chimique et à une aggravation des ulcères de décubitus.

2. Ne pas utiliser d'antipsychotiques en première intention pour traiter les symptômes comportementaux et psychologiques de la démence.

Les personnes atteintes de démence font souvent preuve d'agressivité, de résistance aux soins et d'autres comportements problématiques ou perturbateurs. Dans de telles circonstances, les médicaments antipsychotiques sont souvent prescrits, mais ils présentent des avantages limités et variables tout en présentant des risques, tels qu'une sédation excessive, une dégradation cognitive et un risque accru de chute, d'accident vasculaire cérébral et de mortalité. L’usage de ces médicaments chez des patients atteints de démence devrait être limité aux cas où des mesures non pharmacologiques ont échoué et où les patients représentent une menace imminente pour eux-mêmes ou pour les autres. L'identification et le traitement des causes de changement de comportement peuvent éviter les traitements médicamenteux.

\section{Large soutien et nouvel élan pour \\ la campagne smarter medicine}

Une nouvelle association de soutien dynamise en Suisse l'initiative smarter medicine lancée il y a trois ans. Les orientations de la campagne sont soutenues par des organisations médicales spécialisées et professionnelles, mais aussi par des associations qui défendent les intérêts des patients et des consommateurs. Ensemble, elles souhaitent sensibiliser le public au fait qu'en médecine et pour certains traitements, moins de traitements peut parfois signifier plus de qualité de vie pour les personnes concernées.

Smarter medicine s'est adaptée à I'initiative américaine "Choosing Wisely" qui remporte un grand succès et qui a pour but non seulement de déboucher sur des "décisions judicieuses», mais aussi d'encourager une franche discussion entre le corps médical, les patients et le public. Dans les prochains mois différentes sociétés médicales publieront leurs listes Top- 5 avec des recommandations pour chaque domaine d'activité respectifs. En prélude les prochaines listes concerneront la médecine intensive et la gériatrie. II existe déjà deux listes pour la médecine interne (ambulatoire et stationnaire).

Vous trouvez des informations détaillées concernant I'association de soutien et les listes Top-5 publiées sous www.smartermedicine.ch 
3. Eviter d'utiliser d'autres médicaments que la metformine pour atteindre un niveau d'hémoglobine Alc (HbA1c) inférieur à 7,5\% chez la plupart des personnes âgées; un contrôle modéré de la glycémie est généralement préférable.

Il n'existe aucune preuve que l'usage de médicaments pour obtenir un contrôle glycémique strict est bénéfique chez la plupart des personnes âgées présentant un diabète de type 2 . Chez les adultes n'entrant pas dans la catégorie des personnes âgées, en dehors de la réduction à long terme du risque d'infarctus du myocarde et de mortalité sous metformine, l'usage de médicaments pour abaisser l'hémoglobine glyquée à moins de 7,0\% peut être délétère, y compris un taux de mortalité accru. Chez les personnes âgées, un contrôle strict a systématiquement provoqué des taux plus élevés d'hypoglycémie. Compte tenu des délais importants nécessaires pour atteindre les avantages microvasculaires théoriques d'un contrôle strict, les objectifs glycémiques devraient refléter les objectifs du patient, son état de santé et son espérance de vie. Des objectifs glycémiques raisonnables se situent entre 7,0 et 7,5\% pour les personnes âgées en bonne santé présentant une espérance de vie longue, entre 7,5 et $8,0 \%$ pour celles présentant une comorbidité modérée et une espérance de vie de moins de dix ans, et entre 8,0 et 9,0\% pour celles présentant de multiples comorbidités et une espérance de vie plus courte.

4. Ne pas faire usage de benzodiazépines ou d'autres hypnotiques sédatifs chez les personnes âgées en première intention pour le traitement de l'insomnie, de l'agitation ou du délire.

Des études à grande échelle montrent systématiquement que le risque d'accidents lors de la conduite de véhicules à moteur, de chutes et de fractures de la hanche entraînant une hospitalisation ou le décès du patient peut plus que doubler chez les personnes âgées prenant des benzodiazépines et d'autres hypnotiques sédatifs. Les patients âgés, leurs soignants et leurs prestataires devraient reconnaître cette nocivité potentielle au moment d'opter pour des stratégies de traitement de l'insomnie, de l'agitation ou du délire. L'usage des benzodiazépines devrait être réservé aux symptômes de sevrage alcoolique, au delirium tremens ou

\section{Elaboration de cette liste}

L'American Geriatrics Society (AGS) a mis en place un groupe de travail sous la direction du vice-président du Clinical Practice and Models of Care Committee (CPMC). Les membres du groupe de travail ont été choisis au sein de ce comité, ainsi qu'au sein de I'Ethics Committee, de I'Ethnogeriatrics Committee et du Quality and Performance Measurement Committee (QPMC). Par le biais d'un sondage électronique, les membres de I'AGS ont été invités à donner leur avis et leurs recommandations sur ce qui devrait selon eux figurer sur la liste. Le groupe de travail a d'abord réduit la liste aux dix tests ou méthodes potentiels principaux. II a ensuite examiné les preuves et sollicité l'avis d'experts pour réduire la liste à cinq recommandations qui ont été passées en revue et approuvées par le comité exécutif de l'AGS et par les présidents et vice-présidents du CPMC, de I'Ethics Committee et du QPMC.

Le Comité de la Société professionnelle suisse de gériatrie (SPSG) a examiné les recommandations de la société américaine de gériatrie et a conclu que celles-ci étaient pertinentes et applicables au contexte suisse.

Une liste détaillée des références et des recommandations d'autres sociétés médicales sont disponibles en ligne est sous www.smartermedicine.ch

aux cas de trouble d'anxiété généralisée sévère ne répondant pas aux autres traitements.

5. Ne pas utiliser d'antimicrobiens pour traiter une bactériurie chez des personnes âgées à moins que d'autres symptômes spécifiques des voies urinaires n'aient été constatés.

Des études de cohorte n'ont décelé aucune conséquence défavorable associée à la bactériurie asymptomatique chez les personnes âgées. Les études sur les traitements antimicrobiens de la bactériurie asymptomatique chez les personnes âgées ne démontrent aucun avantage et une augmentation des effets secondaires liés aux antimicrobiens. Des critères de consensus ont été développés pour caractériser les symptômes cliniques spécifiques qui, associés à la bactériurie, définissent une infection des voies urinaires. Le dépistage et le traitement de la bactériurie asymptomatique sont recommandés avant toute instrumentation urologique susceptible de provoquer un saignement muqueux. 